\title{
Three-Layered Design of Electrothermal Actuators for Minimal Voltage Operation
}

\author{
Gal Tibi, ${ }^{1}$ Ela Sachyani Keneth, ${ }^{2}$ Michael Layani, ${ }^{2}$ Shlomo Magdassi, ${ }^{2}$ and Amir Degani ${ }^{1,3}$
}

\begin{abstract}
By designing an actuator composed of thin layers with different coefficients of thermal expansion (CTE) together with an electrically conductive layer, the CTE mismatch can be utilized to produce soft electrothermal actuators (ETAs). These actuators have been typically implemented using only two layers, commonly relying on Timoshenko's analytic model that correlates the temperature to the actuator's curvature. In this study, we extend the analytic model to include the thermoelectric relation present in ETAs, that is, the conductive layer's properties with respect to the operation temperature. By applying the thermoelectric relation, a minimal voltage optimization can be applied to the analytic model. Using dimensionless analysis, we optimize the ETAs performance for both bi- and tri-layer ETAs with and without the thermal modeling. The bi-layer optimization not only predicts the maximal value for the bi-layer performance but also provides the optimal thickness of each layer for any couple of materials. We validate the tri-layer analytic model experimentally by measuring the curvature for different third layer thicknesses. Finally, we optimize the tri-layer design based on the analytic model, which can achieve an improvement in curvature per voltage of $>3000 \%$ over the optimal bi-layer ETA.
\end{abstract}

Keywords: electrothermal actuators, minimal voltage actuation, thermal expansion actuation, soft actuators

\section{Introduction and Related Work}

C Ontinum robots, unlike traditional robots, use a flexible backbone and intrinsic actuation, which spread along the robot's structure instead of rigid links and local actuators. ${ }^{1,2}$ These robots typically maneuver by bending through a series of smooth arcs, such as elephant trunks or tentacles. Continuum robots, by design, can achieve complex configurations of their entire structure. Such hyper-redundant configuration changing capability can be useful in different tasks such as obstacle avoidance or whole arm grasping and manipulation. ${ }^{1,3}$ Owing to their soft structure, these robots benefits from the well-known advantages of soft robotics, that is, to grasp gentle odd-shaped objects without the need of precise position control. ${ }^{4,5}$ However, as in most soft robotic implementations they are known to be difficult to design, control, and sense. ${ }^{6}$

Very thin structures, due to their small area moment of inertia, are inertly soft and bend easily regardless of their composite material elasticity. A wide and thin structure can be tough to bend in one direction and easy in the other. When the thin surface is folded, the resulted structure can be soft or tough depending on the manipulation direction and the structure formation. Activating such thin structures and controlling their shape can potentially revolutionize the soft robotics field. One notable type of robot that utilizes thin structure is origamishaped robots. Origami robots utilize origami folds to morph their shape dramatically. Different configurations can change the structural properties without changing the material properties. Recently, researchers have become interested in the use of flat continuous actuators to achieve desired folding patterns. $^{7,8}$ When actuating a surface continuously, one can control its form and effectively fold the surface as desired. Developing analytic and approximate models of the actuator can help design and control these types of robots.

There are several soft continuum actuation methods in development ${ }^{9}$ such as variable length tendons, ${ }^{10}$ pneumatic, ${ }^{11-13}$ and chemical actuation. ${ }^{14}$ One notable subfield in continuum soft actuators is thin sheet-like robots. Unlike the earlier actuating methods, these robots do not inherit their softness from material properties but from their slender thickness. These robots

\footnotetext{
${ }^{1}$ Technion Autonomous Systems Program, Technion Israel Institute of Technology, Haifa, Israel.

${ }^{2}$ Casali Center of Applied Chemistry, Institute of Chemistry and the Center for Nanoscience and Nanotechnology, The Hebrew University of Jerusalem, Jerusalem, Israel.

${ }^{3}$ Department of Environmental, Water and Agricultural Engineering, Faculty of Civil and Environmental Engineering, Technion Israel Institute of Technology, Haifa, Israel.
} 
are usually manipulated by utilizing a complex material property to create a specific strain pattern. There are several actuating principles used for these sheet-like robots. One example is electroactive polymers (EAPs), where strain is created by applying electrical power. EAPs are typically classified into two dominant groups based on their operating principles, namely, dielectric and ionic. Dielectric EAPs utilize electrostatic, piezoelectric, or ferroelectric forces that squeeze a polymer between two surfaces. Dielectric EAPs are characterized with fast response but require high voltages $\left(\sim 200 \mathrm{MVm}^{-1}\right)$ to operate. ${ }^{15}$ Ionic EAPs are based on mobility or diffusion of ions, ${ }^{16}$ they require low voltages $(\sim 5 \mathrm{~V}),{ }^{15}$ but they have to be submerged in an electrolyte solution to operate. Other sheet-like robotic actuation includes shape memory materials ${ }^{17}$ and liquid absorption, where water-based ink is printed onto paper causing a swelling that forces the paper to bend. ${ }^{18,19}$

Recently, another type of EAP has been receiving more research attention, electrothermal actuators (ETAs). ETAs are usually based on either thermal expansion of a single film ${ }^{20-22}$ or on the difference in thermal expansion between two layers. ${ }^{4,23-29}$ Some ETAs can be printed, making the prototyping relatively cheap and available for researchers. Unlike other EAPs, ETAs can be activated under relatively low voltage and do not require to be submerged in fluids, making them promising for practical use in soft sheet-like robots. A few of our initial prototypes of soft sheet-like robots are shown in Figure 1. This article will not focus on the macro design of such robots but will investigate the design of a single module, such as the materials optimal properties and thicknesses of the layers.

Bi-layer ETAs are similar in principle to thermostats based on the bi-metal effect. The bi-metal effect occurs when materials with a different coefficient of thermal expansion (CTE) are bonded together and a change in temperature is applied. When a single layer undergoes a temperature change, the layer strains according to its CTE. However, when a multiple layer structure undergoes a temperature change the boundary constraint between the layers forces the entire structure to bend. Although thermal expansion causes only small strains, typically a few dozen microstrains, the structure's curvature and deflection can be substantial. This bi-metal effect was first analytically modeled by Timoshenko ${ }^{30}$ in the 1920 s and has been widely used in the industry for the design of thermostats and thermometers. ${ }^{31}$
The popularity of bi-metal temperature sensors is mostly due to the linear relation between the curvature of the bi-layer beam to the change of temperature and due to their inexpensive manufacturing process. The bi-layer actuator can achieve remarkable results. Researchers have managed to construct an inchworm robot that can lift almost seven times its own weight ${ }^{26}$ and a twistable arm that can lift 26 times its own weight. ${ }^{3}$ However, although a reliable analytic model is available, most bi-layer actuators are designed by the general guideline of choosing materials with the highest CTE difference while ignoring the effects of the stiffness and thickness of each layer. ${ }^{23}$

The analytic model for a multilayer thermally deforming structure was established in the 1960s for the production of more sensitive thermostats. ${ }^{32}$ More recently, the model was investigated for the three-layered (tri-layer) case with the intention of reducing layer delamination. By adding a third layer the location of the neutral plane, where the elongation and stresses are zero, can be shifted toward the problematic layer bond location without loss of the bi-layer performance. ${ }^{33}$ Unfortunately, thermally activated tri-layer actuators could not improve upon the bi-layer's curvature sensitivity to temperature. ${ }^{34,35}$ However, the ETA, due to its electric actuation has a temperature-to-thickness coupling and, therefore, can benefit from a tri-layer design. The objective of this study is to reconsider the tri-layer design for ETA design while taking into account the temperature-to-thickness relation.

In our previous studies, ${ }^{36,37}$ we introduced a thermal model to include the relation between the temperature and the conductive layer's thickness (for both bi-layer and tri-layer). We conducted experiments to measure the curvature for different third layer thicknesses. The experiments' results were similar to the analytic model's theoretical curvature. Furthermore, the tri-layer ETA had demonstrated a significant improvement in performance, that is, achieving better curvature per voltage, over the bi-layer ETA design. These promising experimental results had motivated us to search for the optimal tri-layer parameters.

In this article, we begin by using dimensionless analysis to optimize the bi-layer actuator analytic model with and without the thermal modeling. The bi-layer optimization not only predicts the maximal value for the bi-layer performance but also provides the optimal thickness of each layer for any couple of materials. We reproduce the former tri-layer

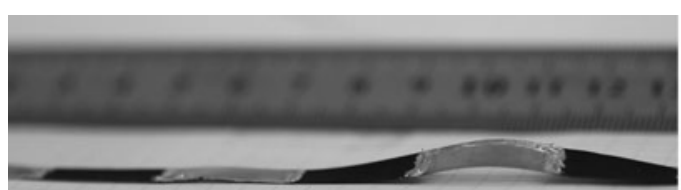

a

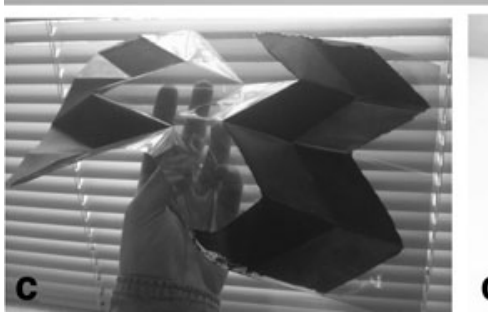

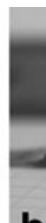

b

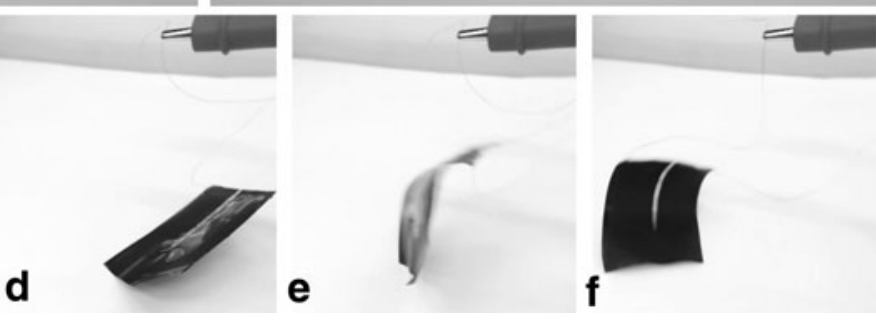

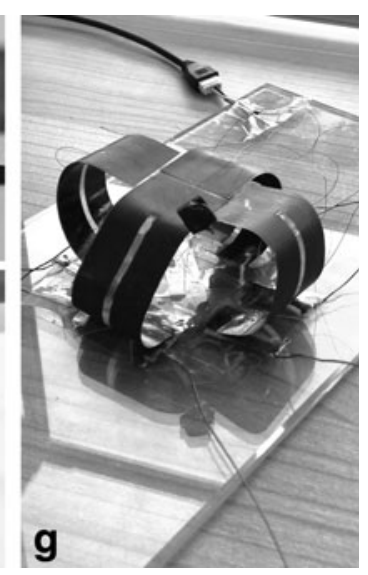

FIG. 1. Four of our initial soft sheet-like robot prototypes actuated by an electrothermal actuator. (a, b) A multisegment snake-like robot in two different configurations. (c) An origami robot prototype based on the Miura-ori origami pattern. (d-f) A single segment actuator under actuation from the initial state (d) to the final actuated state (f). (g) A four-segment parallel robot carrying a small video camera and controlling its point-of-view orientation. 
analytical model while including the thermoelectric relation by applying, in our opinion, a more intuitive and lower dimensional parameterization. We validate the tri-layer model experimentally based on our prior experiments ${ }^{37}$ by applying data analysis to better handle tolerances in the experiment. Finally, we optimize the tri-layer design based on the analytic model and achieve a significant improvement of $>3000 \%$ over the best possible electrically powered bi-layer actuator.

\section{Bi-Layer Modeling and Optimization}

We begin by analyzing the bi-layer mechanism to better understand the principles behind the tri-layer actuator and to compare the tri-layer actuator performance with the bi-layer results.

\section{Bi-layer modeling}

When two materials with a different CTE are bounded together, a change of temperature will cause each material to expand differently and will, therefore, cause the structure to bend. Timoshenko formulated the two-layer model for a bimetal beam. ${ }^{30}$ This model has become an industry standard in bi-metal thermostat design. ${ }^{31}$ The model describes the relation between the beam's curvature, $\kappa$, to the layer's dimensions, and material properties. The model assumes the following:

1. Layers behave as beams capable of axial and bending deformations.

2. The beam curves around a single axis.

3. The deformation is linear.

4. No slip occurs at the interface of the two beams.

5. Constant material properties throughout each layer.

6. Uniform temperature throughout the beam.

Timoshenko's model was meant for thermal sensor design, such as the bimetallic strip thermostat. These sensors curve under temperature change caused by an external heat source. However, in ETA design, the heat source is internal and is a function of the actuator's parameters. We distinct between two heating cases: external heating, such as thermostats, where the actuator's temperature is decoupled from its design, and internal heating, such as ETAs, where the heat source is integrated within the actuator and couples the temperature with the design parameters. We will next apply a thermoelectric model to Timoshenko's model to fit the external heating model to the internal heating ETA design problem, achieving the highest deflection for a given voltage. Besides achieving the highest deflection, we also aim to use low voltage since we intend to use these actuators on batteryoperated robots.

Our basic actuator model, as depicted in Figure 2, is two bonded rectangular beams with length $l$, width $w$, each layer with thicknesses $t_{1}, t_{2}$, elasticity moduli $E_{1}, E_{2}$, and CTE $\alpha_{1}, \alpha_{2}$. The actuator is heated by $\Delta T$ degrees. By applying Timoshenko's formula, we arrive at the thermomechanical relationship

$$
\kappa=2\left(\alpha_{1}-\alpha_{2}\right) \frac{\left(t_{1}+t_{2}\right)}{\left(t_{1}+t_{2}\right)^{2}+\frac{1}{3}\left(E_{1} t_{1}^{3}+E_{2} t_{2}^{3}\right)\left(\frac{1}{E_{1} t_{1}}+\frac{1}{E_{2} t_{2}}\right)} \cdot \Delta T .
$$

Since we are interested in designing a thermoelectric actuator, we will need to evaluate the temperature to electrical

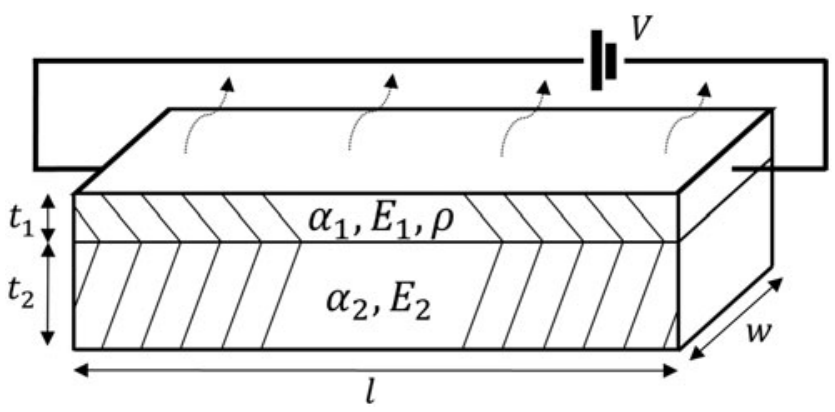

FIG. 2. Schematics of the bi-layer actuator. Adapted from Ref. $^{36}$

input relation. At steady state, the power input is equal to the power output. Since the output heat flux, in our expected temperature range, is mostly by convection, the temperature difference $(\Delta T)$ is equal to the power input multiplied by a constant, $C$. The actuator utilizes one of its layers (in our example, layer 1) as an electrical resistor to produce the actuating internal heat. The electrical power is linear to the voltage $(V)$ squared, and is inversely proportional to the circuit resistance $R$. The resistance is proportional to the dimensions and the material's resistivity $\rho$. Hence, using assumption VI from earlier, we arrive at the thermoelectric relationship

$$
\Delta T=C \cdot \text { Power }=C \frac{V^{2}}{R}=C \frac{V^{2} w t_{1}}{\rho l} .
$$

Substituting the thermoelectric relationship [Equation (2)], into the thermomechanical relationship [Equation (1)], we arrive at the next equation describing the combined electromechanic curvature equation

$$
\begin{aligned}
\Delta \kappa= & \frac{C V^{2}}{\rho} \cdot\left(\alpha_{1}-\alpha_{2}\right) \cdot \frac{w}{l} \\
& \cdot \frac{2 t_{1}\left(t_{1}+t_{2}\right)}{\left(t_{1}+t_{2}\right)^{2}+\frac{1}{3}\left(E_{1} t_{1}^{3}+E_{2} t_{2}^{3}\right)\left(\frac{1}{E_{1} t_{1}}+\frac{1}{E_{2} t_{2}}\right)} .
\end{aligned}
$$

For each of the aforementioned cases, external and internal heating, we would like to maximize sensitivity to its input, either to temperature change or to voltage change. To do so, we rewrite [Equations (1) and (3)] using dimensionless parameterization. Choosing the dimensionless ratios $t_{21}=t_{2} / t_{1}$, $E_{21}=E_{2} / E_{1}$, we arrive at

$$
\begin{aligned}
\kappa_{E x t}= & \frac{1}{t_{1}} \cdot \Delta T \cdot\left(\alpha_{2}-\alpha_{1}\right) \\
& \cdot \frac{2\left(1+t_{21}\right)}{\left(1+t_{21}\right)^{2}+\frac{1}{3}\left(1+E_{21} t_{21}{ }^{3}\right)\left(1+\frac{1}{t_{21} E_{21}}\right)}, \\
\kappa_{I n t}= & \frac{C V^{2}}{\rho} \cdot \frac{w}{l} \cdot\left(\alpha_{2}-\alpha_{1}\right) \\
& \cdot \frac{2\left(1+t_{12}\right)}{\left(1+t_{21}\right)^{2}+\frac{1}{3}\left(1+E_{21} t_{21}{ }^{3}\right)\left(1+\frac{1}{t_{21} E_{21}}\right)},
\end{aligned}
$$


where $\kappa_{\text {Ext }}$ is the curvature in the external heating (sensor) case and $\kappa_{\text {Int }}$ is the coupled ETAs internal heating curvature.

\section{Bi-layer optimization}

As mentioned earlier, we distinct between the two heating cases, external and internal in Equation (4). In both cases, choosing a material couple with a high difference in CTE, $\left(\alpha_{2}\right.$ $-\alpha_{1}$ ), will linearly increase the actuator's sensitivity. The second common factor between the two models in Equation (4) is the rightmost "design factor," which only depends on the two dimensionless parameters of thickness $\left(t_{21}\right)$ and elasticity $\left(E_{21}\right)$. We can analytically find the optimal $t_{21}, E_{21}$ that will bring this design factor to a maximal value. To maximize the design factor term, the optimal relation between the two dimensionless parameters should be $E_{21_{\text {opt bi }}}=\left(1 / t_{12}\right)^{2}$. After substituting $E_{21}$ with $E_{21 \text { opt bi }}$ the bi-layer design factor in Equation (4) simplifies to $1.5 /\left(t_{21}+1\right)$. Since the thickness ratio $t_{21}$ is positive, the smaller the thickness ratio, the larger the design factor, with a maximal value of 1.5 . We can then substitute the optimal design factors into Equation (4) to analyze the complete curvature expression for both cases. The optimal curvatures are then simplified to

$$
\begin{aligned}
& \kappa_{o p t b i \text { Ext }}=\Delta T \cdot \frac{3\left(\alpha_{2}-\alpha_{1}\right)}{2\left(t_{1}+t_{2}\right)}, \\
& \kappa_{o p t \text { bi Int }}=\frac{C V^{2}}{\rho} \cdot \frac{w}{l} \cdot \frac{3\left(\alpha_{2}-\alpha_{1}\right)}{2\left(t_{21}+1\right)} .
\end{aligned}
$$

It is important to notice how these two optimal cases differ. The external case is inversely proportional to the total structure thickness $\left(t_{1}+t_{2}\right)$, whereas the internal case is inversely proportional to the thickness ratio $\left(t_{21}+1\right)$. Both the thickness and the thickness ratio are bounded by production capabilities. However, the external case could, without manufacturing constraints, infinity amplify its temperature sensitivity. In the internal heating case, voltage sensitivity is bounded by 1.5 since the thickness ratio cannot be negative. The difference in possible sensitivity is due to the conflicting demands: the structure should be not only as thin as possible but also conduct electricity well. The objective of this study is to solve this issue. By adding a third layer we can bypass this problem as will be demonstrate in the following sections.

We are left with optimizing the design parameters $w, l$, and the conductive layer resistivity $\rho$, where the optimization is trivial as the sensitivity to $V^{2}$ is linear to $w \cdot(l \cdot \rho)^{-1}$. There are, of course, many more complicated reasons to choose a certain material for each layer, such as heat endurance, manufacturing capabilities, and cost. The aforementioned criteria has been discussed in previous studies, and good material candidates for each of the layers were suggested (e.g., in Ref. ${ }^{38}$ ). For different materials, more complicated design issues may arise. For example, the change in material properties, such as elasticity and resistivity, may change as a function of temperature and should be addressed according to the materials and range of temperatures. Another issue involves the fact that the actuator might not only curve around a single axis hence, a wide actuator will not behave according to the aforementioned model since it will not comply with assumption II.

\section{Tri-Layer Modeling and Optimization}

Even though bi-layer ETAs can achieve fair performance, in the Bi-layer optimization section we showed how internal heating limits the actuator's performance. The goal of this study is to show how adding a third layer can alleviate part of these limitations. Tri-layer actuators have already been shown to achieve better traits such as higher actuation force and lower buckling temperature. ${ }^{32,39}$ In our previous study, ${ }^{37}$ we have also shown how, in the electrically driven case, the tri-layer design can achieve higher curvature sensitivity to voltage. By adding a third layer, however, the analysis and design becomes more complicated. In this section, we will develop the analytic curvature model while including the thermoelectric relation, followed by the model validation experiments, and finally the optimal parameter choices.

\section{Tri-layer analytic model}

Vasudevan and Johnson ${ }^{32}$ used Timoshenko's bi-metal approach $^{30}$ to develop the thermomechanical relation of a more general multilayer model. Based on their model, they have found that the tri-layer beam could outperform the bilayer as a temperature sensor by lowering the buckling temperature. Since we are interested in the performance of an actuator, that is, internal heating ETA, we modified the model to include the thermoelectric relation, Equation (2), as we have done in the bi-layer case in Equation (3). In this section, we develop the analytic model using different parameterization to achieve better understanding of the tri-layer performance. We compare the theoretical bi- and tri-layer performance to see if the better performance expected of the tri-layer sensor will also appear in the tri-layer actuator (ETA), where the temperature is parameter dependent, as shown in Equation (2).

To model the tri-layer actuator, shown in Figure 3, we use the same geometric parameters and assumptions as for the bi-layer modeling, where $E_{3}, t_{3}, \alpha_{3}$ are the parameters of a third layer. We first write the expression for the linear elongation, $\varepsilon$, along the $x$ coordinate as a function of the $z$ coordinate as $\varepsilon(z)=\varepsilon_{0}+\kappa \cdot z$, where $\varepsilon_{0}$ is the elongation at $z=0$. We can express the force and moment equilibrium equations as a function of the material elongations while accounting for the thermal expansion. The force equilibrium about the $x$ axis is

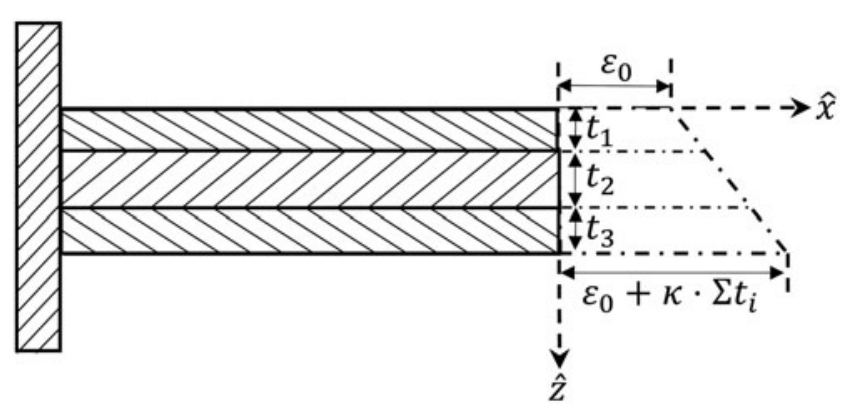

FIG. 3. A schematic view of the mounted tri-layer cross section. The linear elongation along the $x$ axis is as a function of the bias elongation at $z=0, \varepsilon_{0}$ and the beam's curvature $\kappa$. 


$$
\begin{gathered}
\int_{0}^{t_{1}} w E_{1}\left(\varepsilon_{0}+\kappa z\right) d z+\int_{t_{1}}^{t_{1}+t_{2}} w E_{2}\left(\varepsilon_{0}+\kappa z\right) d z+\int_{t_{1}+t_{2}}^{t_{1}+t_{2}+t_{3}} w E_{3}\left(\varepsilon_{0}+\kappa z\right) d z=\int_{0}^{t_{1}} w E_{1} \alpha_{1} \Delta T d z \\
+\int_{t_{1}}^{t_{1}+t_{2}} w E_{2} \alpha_{2} \Delta T d z+\int_{t_{1}+t_{2}}^{t_{1}+t_{2}+t_{3}} w E_{3} \alpha_{3} \Delta T d z
\end{gathered}
$$

and the moment equilibrium around $z=0, x=0$

$$
\begin{gathered}
\int_{0}^{t_{1}} w E_{1}\left(\varepsilon_{0}+\kappa z\right) z d z+\int_{t_{1}}^{t_{1}+t_{2}} w E_{2}\left(\varepsilon_{0}+\kappa z\right) z d z+\int_{t_{1}+t_{2}}^{t_{1}+t_{2}+t_{3}} w E_{3}\left(\varepsilon_{0}+\kappa z\right) z d z=\int_{0}^{t_{1}} w E_{1} \alpha_{1} \Delta T z d z \\
+\int_{t_{1}}^{t_{1}+t_{2}} w E_{2} \alpha_{2} \Delta T z d z+\int_{t_{1}+t_{2}}^{t_{1}+t_{2}+t_{3}} w E_{3} \alpha_{3} \Delta T z d z .
\end{gathered}
$$

Equations (6) and (7) can be written in matrix form as

$$
\begin{aligned}
& \left(\begin{array}{lc}
\frac{E_{1} t_{1}^{2}+E_{2}\left(\left(t_{1}+t_{2}\right)^{2}-t_{1}^{2}\right)+E_{3}\left(\left(t_{1}+t_{2}+t_{3}\right)^{2}-\left(t_{1}+t_{2}\right)^{2}\right)}{2} & E_{1} t_{1}+E_{2} t_{2}+E_{3} t_{3} \\
\frac{E_{1} t_{1}^{3}+E_{2}\left(\left(t_{1}+t_{2}\right)^{3}-t_{1}^{3}\right)+E_{3}\left(\left(t_{1}+t_{2}+t_{3}\right)^{3}-\left(t_{1}+t_{2}\right)^{3}\right)}{3} & \frac{E_{1} t_{1}^{2}+E_{2}\left(\left(t_{1}+t_{2}\right)^{2}-t_{1}^{2}\right)+E_{3}\left(\left(t_{1}+t_{2}+t_{3}\right)^{2}-\left(t_{1}+t_{2}\right)^{2}\right)}{2}
\end{array}\right)\left(\begin{array}{c}
\kappa \\
\varepsilon_{0}
\end{array}\right) \\
& =\Delta T\left(\begin{array}{c}
t_{1} \alpha_{1}+t_{2} \alpha_{2}+t_{3} \alpha_{3} \\
E_{1} \frac{t_{1}^{2}}{2} \alpha_{1}+E_{2} t_{2}\left(t_{1}+\frac{t_{2}}{2}\right) \alpha_{2}+E_{3} t_{3}\left(t_{1}+t_{2}+\frac{t_{3}}{2}\right) \alpha_{3}
\end{array}\right) .
\end{aligned}
$$

By solving this system of equations, we can explicitly find the curvature $(\kappa)$ and bias $\left(\varepsilon_{0}\right)$

$$
\begin{gathered}
\kappa=\frac{\Delta T \cdot 2\left(\frac{\left(\alpha_{1}-\alpha_{2}\right)\left(t_{1}+t_{2}\right)}{t_{3} E_{3}}+\frac{\left(\alpha_{2}-\alpha_{3}\right)\left(t_{2}+t_{3}\right)}{t_{1} E_{1}}+\frac{\left(\alpha_{1}-\alpha_{3}\right)\left(t_{1}+2 t_{2}+t_{3}\right)}{t_{2} E_{2}}\right)}{\frac{\left(t_{2}+t_{3}\right)^{2}}{E_{1} t_{1}}+\frac{\left(t_{1}+2 t_{2}+t_{3}\right)^{2}}{E_{2} t_{2}}+\frac{\left(t_{1}+t_{2}\right)^{2}}{E_{3} t_{3}}+\frac{\left(E_{1} t_{1}^{3}+E_{2} t_{2}^{3}+E_{3} t_{3}^{3}\right)\left(E_{1} t_{1}+E_{2} t_{2}+E_{3} t_{3}\right)}{3 E_{1} E_{2} E_{3} t_{1} t_{2} t_{3}}}, \\
\varepsilon_{0}=\Delta T \cdot\left(\begin{array}{c}
\frac{\alpha_{2} t_{3}^{2}+3 \alpha_{2}\left(t_{2}+t_{3}\right)^{2}+\alpha_{3} t_{2}^{2}}{3 E_{1} t_{1}}+\frac{\alpha_{1}\left(4 t_{3}^{2}+3 t_{1} t_{3}\right)+\alpha_{3}\left(4 t_{1}^{2}+3 t_{1} t_{3}\right)}{3 E_{2} t_{2}}+\ldots \\
\frac{\alpha_{1}\left(4 t_{2}^{2}+3 t_{1}^{2}\right)+3 \alpha_{2}\left(t_{1}+t_{2}\right)^{2}}{3 E_{3} t_{3}}+\frac{\alpha_{1}\left(t_{1}+2 t_{2}+3 t_{3}\right)+\alpha_{3}\left(3 t_{1}+2 t_{2}+t_{3}\right)}{E_{2}}+\frac{E_{1}^{2} t_{1}^{4} \alpha_{1}+E_{2}^{2} t_{2}^{4} \alpha_{2}+E_{3}^{2} t_{3}^{4} \alpha_{3}}{3 E_{1} E_{2} E_{3} t_{1} t_{2} t_{3}}
\end{array}\right) / \\
\left(\frac{\left(t_{2}+t_{3}\right)^{2}}{E_{1} t_{1}}+\frac{\left(t_{1}+2 t_{2}+t_{3}\right)^{2}}{E_{2} t_{2}}+\frac{\left(t_{1}+t_{2}\right)^{2}}{E_{3} t_{3}}+\frac{\left(E_{1} t_{1}^{3}+E_{2} t_{2}^{3}+E_{3} t_{3}^{3}\right)\left(E_{1} t_{1}+E_{2} t_{2}+E_{3} t_{3}\right)}{3 E_{1} E_{2} E_{3} t_{1} t_{2} t_{3}}\right) .
\end{gathered}
$$

We can then apply the same thermoelectric model as in Equation (2) to the tri-layer curvature model in Equation (8) to arrive at the electro-mechanical relationship of the ETA

$$
\kappa=\frac{C V^{2}}{\rho} \cdot \frac{w}{l} \cdot \frac{2 t_{i}\left(\frac{\left(\alpha_{1}-\alpha_{2}\right)\left(t_{1}+t_{2}\right)}{t_{3} E_{3}}+\frac{\left(\alpha_{2}-\alpha_{3}\right)\left(t_{2}+t_{3}\right)}{t_{1} E_{1}}+\frac{\left(\alpha_{1}-\alpha_{3}\right)\left(t_{1}+2 t_{2}+t_{3}\right)}{t_{2} E_{2}}\right)}{\frac{\left(t_{1}+t_{2}\right)^{2}}{t_{3} E_{3}}+\frac{\left(t_{1}+2 t_{2}+t_{3}\right)^{2}}{t_{2} E_{2}}+\frac{\left(t_{2}+t_{3}\right)^{2}}{t_{1} E_{1}}+\frac{\left(E_{1} t_{1}^{3}+E_{2} t_{2}^{3}+E_{3} t_{3}^{3}\right)\left(t_{1} E_{1}+t_{2} E_{2}+t_{3} E_{3}\right)}{3 E_{1} E_{2} E_{3} t_{1} t_{2} t_{3}}} .
$$

Here, $t_{i}$ represents the thickness of the conductive layer, which can be any of the three layers, $i \in[1,2,3]$. As in the bi-layer case, both Equations (8) and (10) have a common factor that dictates the actuator's sensitivity to input, which we refer to as the design factor (rightmost term), which is a function of $E_{1,2,3}, \alpha_{1,2,3}, t_{1,2,3}$. One can verify that Equation (10) reduces to the bi-layer curvature expression in Equation (3) as $t_{3} \rightarrow 0$. In the following section, we will experimentally verify this tri-layer analytic model. 
Table 1. Parameters Values of the Three Layers

\begin{tabular}{llllll}
\hline$t_{1}(\mu \mathrm{m})$ & $1.9 \pm 0.3$ & $E_{1}(\mathrm{MPa})$ & $250 \pm 50$ & $\alpha_{1}\left(\mathrm{~K}^{-1}\right)$ & $-15 \pm 5 \times 10^{-6}$ \\
$t_{2}(\mu \mathrm{m})$ & $50 \pm 5$ & $E_{2}(\mathrm{MPa})$ & $1500 \pm 200$ & $\alpha_{2}\left(\mathrm{~K}^{-1}\right)$ & $31.2 \pm 0.5 \times 10^{-6}$ \\
$t_{3}(\mu \mathrm{m})$ & {$[50,100,150,200] \pm 10$} & $E_{3}(\mathrm{MPa})$ & $35 \pm 2$ & $\alpha_{3}\left(\mathrm{~K}^{-1}\right)$ & $189 \pm 1 \times 10^{-6}$ \\
\hline
\end{tabular}

\section{Model verification experiments}

The design factor of the tri-layer analytic model, Equation (8), consists of nine design parameters $\left(E_{1,2,3}, \alpha_{1,2,3}, t_{1,2,3}\right)$ that determine the beam's curvature sensitivity to heat. To simplify matters and to demonstrate the tri-layer's superiority over the bi-layer, we measured the curvature while changing only a single parameter, the thickness of the newly added third layer, $t_{3}$. By keeping the first layer thickness constant in all experiments, we can apply the same voltage on all our samples and reach similar temperatures.

Experiment description and methods. This experiment is identical to our previous experiment in Ref. ${ }^{37}$; however, the fabrication processes are briefly presented here, whereas a full description and material properties measurements results of the actuator fabrication are detailed in our previous report and in the Appendix.

We chose similar materials as were used in other ETAs' designs. ${ }^{3,4,10,19,23-27,38}$ The conductive layer material was chosen to be carbon nanotubes (CNTs) network and the second (middle) layer to be a Kapton polymer. CNT is a low resistivity conductor with low elasticity (in a network form), it has a negative CTE and can be applied with high layer thickness resolution. For the middle layer we used a Kapton polymer, which has a high resilience to heat and relatively high CTE. The third layer material is a flexible polymer made from a ultraviolet (UV) curable ink composed of aliphatic urethane acrylate monomers (CN9900; Sartomer, Belgium, and a photoinitiator (diphenyl(2,4,6-trimethylbenzoyl) phosphine oxide, TPO; BASF, Germany). ${ }^{37}$ We will refer to this third layer material simply as the "third layer." Similar to measurements we previously performed in Ref., ${ }^{37}$ the Kapton's thickness, $t$, and tolerance were given by the manufacturer, all other thicknesses were measured using a DualBeam Focused Ion Beam (FIB)/SEM system (Helios NanoLab 460F1; FEI). Young's elastic moduli, $E$, were measured using an INSTRON 4500 system. The CTE values, $\alpha$, were measured using TA optical dilatometer TI DIL806 806 (TA Instruments). A summary of all parameter values and their uncertainty intervals due to production tolerances and measurement errors are given in Table 1.

Connecting the actuator to a power source without impairing the actuator's movement requires a specialized design. The most common design for mounted ETAs is the U-shaped design. ${ }^{24,38}$ The U-shaped design, as illustrated in Figure 4, has its conductive layer placed similar to the letter "U." In this layout, both electrical connections are on one side, which when mounted, allows the actuator to move freely without disturbances from electrical wires. As shown in Figure 4, the samples were mounted on one side of the actuator and the motion occurred on a plane perpendicular to the ground. Each sample was subjected to a voltage of $75 \mathrm{~V}$ fed through the mount. The samples were recorded using a camera and an FLIR E4 thermal camera. For a given voltage, the temperature was recorded, and the curvature was measured by fitting a circle to the final actuator configuration, as shown in Figure 5 (bottom row). The circle fitting was done by hand while ensuring that the actuator's mounted edge overlaps the circle at the correct angle.

The experiment included testing 12 samples of a bilayer actuator with the same parameters before adding the third layer. Each tested sample was retested with the added third layer. In total, three samples for each third layer thickness value, $t_{3}=[50,100,150,200] \pm 10 \mu \mathrm{m}$, were tested.

The manufacturing process of the actuator may cause an initial curvature of the samples at room temperature. To account for this initial curvature, we calculated the difference between the initial curvature and the actuated state after the sample reached steady state. This correction is appropriate since the curvature has a linear correlation to the temperature change. The maximal temperature of all samples at the same voltage varied between $[73,120]^{\circ} \mathrm{C}$ due to variation in the conductive layer thickness and in the
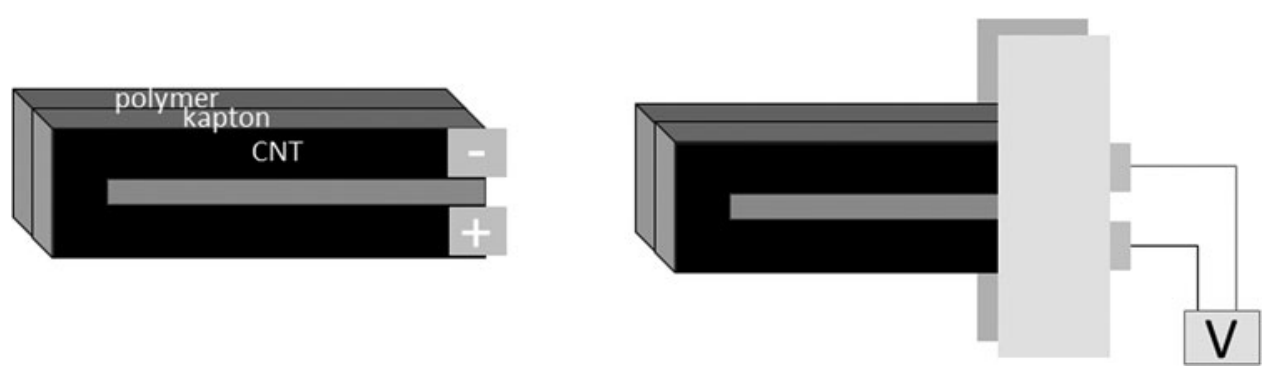

FIG. 4. A schematic of the tri-layer actuator structure, mounting, and electrical connections. Left: The tri-layer electrothermal actuator layer's material in order with the copper tape electrodes illustrated as small rectangles with a plus or minus symbol. Right: The tri-layer electrothermal actuator mounted between two walls while connected to a DC power source. 


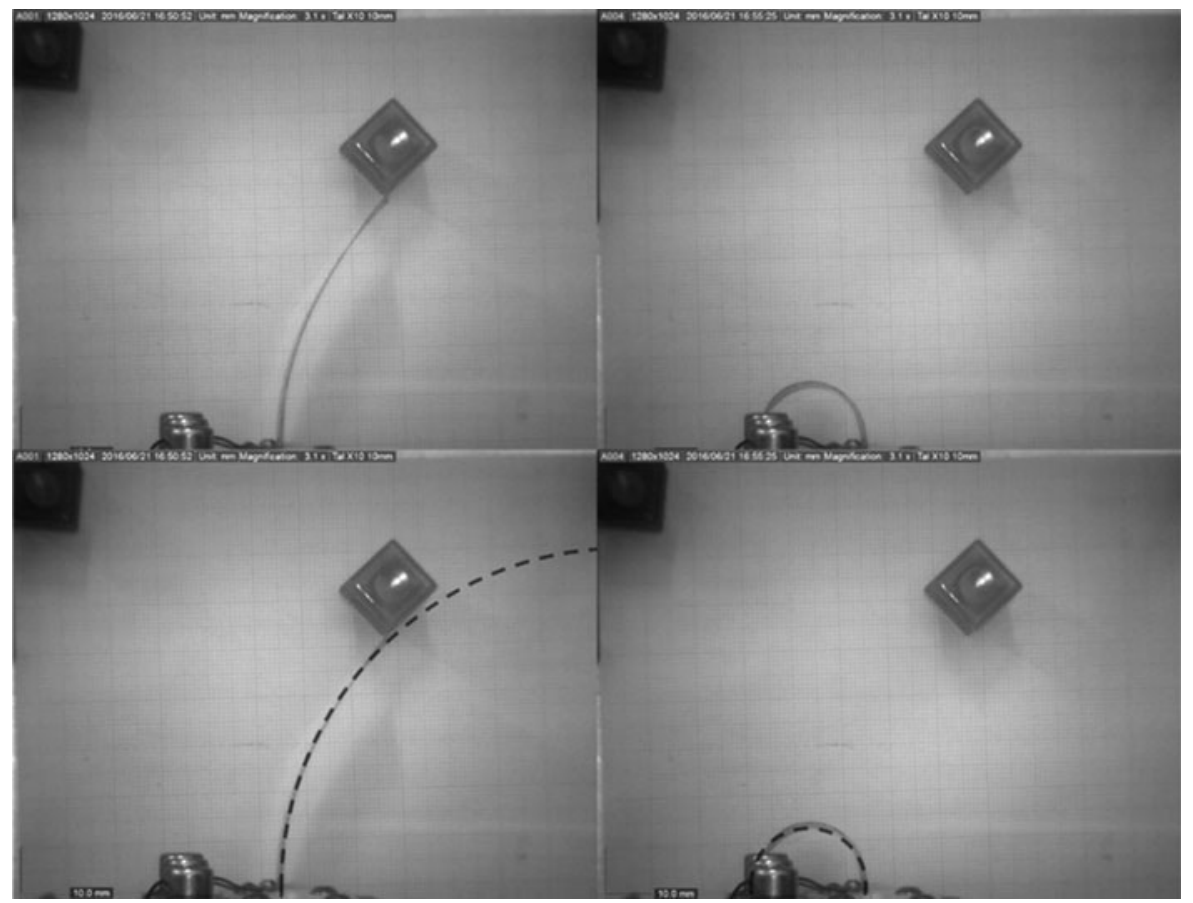

FIG. 5. An example of the actuator response to voltage. The actuator $\left(t_{3}=50 \mu \mathrm{m}\right)$ before applying voltage (left) and at steady state after applying voltage (right). Top row shows the raw images, whereas the bottom row shows the fitting of the circles (dashed black) to measure the radius of curvature.

CNT's electrical conduciveness $\rho .^{37}$ To account for these variations in conductivity, the curvature difference between the initial and final state was normalized by the maximal temperature.

Experiment results. As Figure 6 depicts, the added layer has shown a significant improvement over the tested bi-layer $\left(t_{3}=0\right)$. There also seems so be an optimal third layer thickness between 0 and $150 \mu \mathrm{m}$. Although the CTE of the third layer is only about six times the CTE of the middle layer, the tri-layer achieved $>20$ times the sensitivity of the bi-layer.

Comparing the analytic model to experiments. To compare the analytic model with the experimental results, we plotted, in Figure 7, the experiment results from Figure 6 overlaid with the analytic model in Equation (8). To account for the uncertainty in the parameters, we calculated the trilayer model for randomly chosen values within the uncertainty intervals given in Table 1. As can be seen in Figure 7,

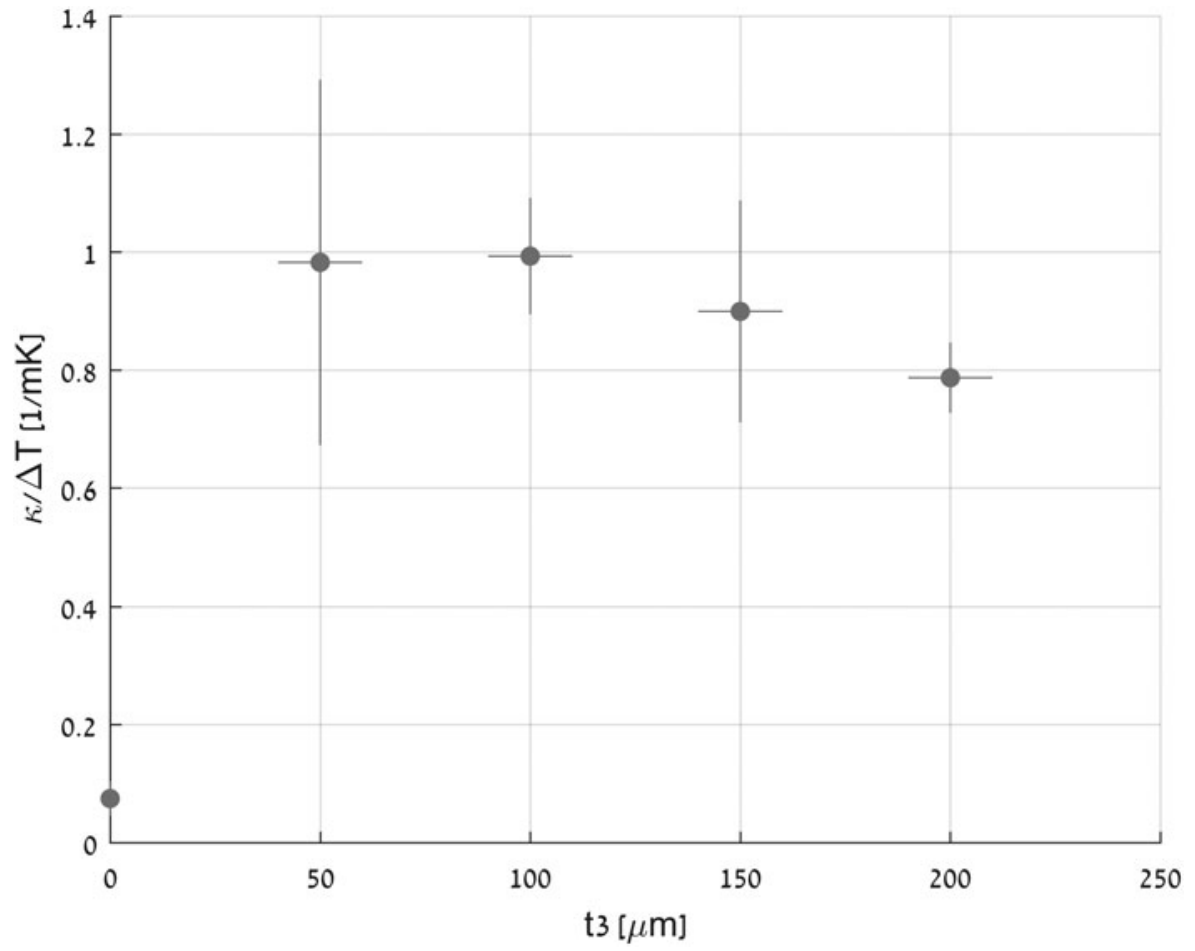

FIG. 6. The beam's curvature at $75 \mathrm{~V}$ normalized by the measured temperature for different third layer thickness. The vertical error bars are the standard deviation and the horizontal error bars are the manufacturing tolerances of the third layer thickness. The data for the bi-layer $\left(t_{3}=0\right)$ were based on 12 specimens and each of the tri-layer's thickness was based on four specimens. 
FIG. 7. Comparison of the experiment results and the analytic simulation. The experiment results (in red) overlaid on with the analytic model prediction as a function of the third layer thickness $t_{3} \in[0,250] \mu \mathrm{m}$. The other parameter values for each of the $10^{6}$ points were randomly chosen from Table 1 to cover all possible parameter values. Color images are available online.

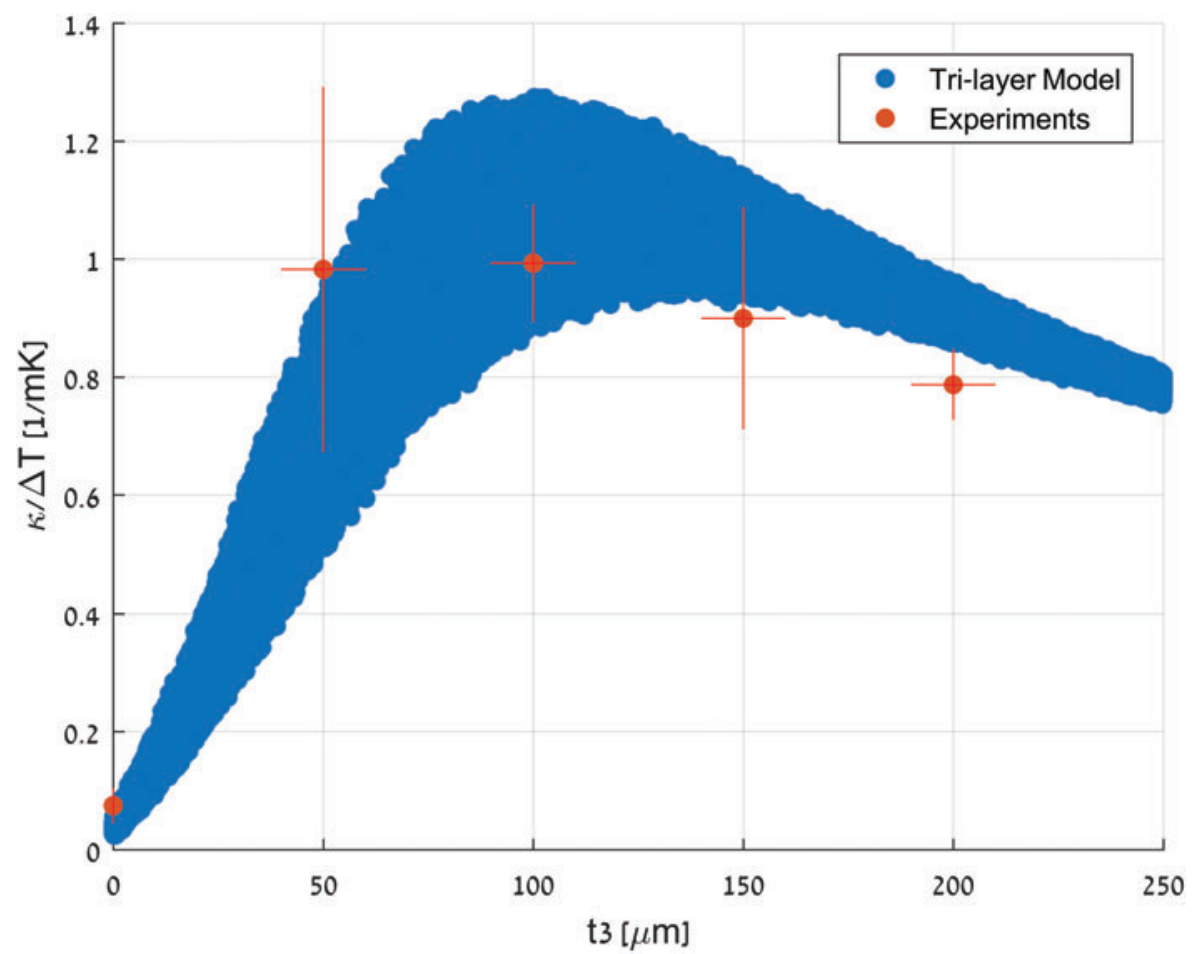

the analytic model had demonstrated a reasonable accuracy by predicting the tri-layer performance as a function of the third layer thickness.

\section{Tri-layer optimization}

As in the bi-layer optimization (Bi-Layer Optimization section), we can divide the optimization into two different heating methods, external and internal. To optimize the trilayer design we will need to maximize the actuator's sensitivity to either temperature or voltage change. The externally heated tri-layer has been previously investigated and was found to achieve similar curvature sensitivity to temperature as the bi-layer. ${ }^{32,34,39}$ However, this is not true for the case of internal heating, that is, when one of the layers is responsible for heating the structure. In this section, we will focus on optimizing the tri-layer sensitivity to voltage in the internal heating case. Unfortunately, due to the addition of parameters, the tri-layer design factor in Equation (8) is more complicated than the bi-layer's design factor in Equation (1). Furthermore, it is impossible to decouple the tri-layer curvature expression to independent factors and separate the CTEs from the design factor as was done in the bi-layer cases, that is, Equations (1) and (3).

To tackle the complexity of the design factor, we applied a numerical approach. First we chose feasible parameter values based on commonly used engineering materials, for example, given in Refs. ${ }^{6}$ and ${ }^{40}$ These intervals are given in Table 2. We randomly picked $10^{9}$ different parameter combination of $t_{1,2,3}, E_{1,2,3}, \alpha_{1,2,3}$ from the intervals in Table 2 , and for each random combination we computed the design factor's numerical value. The number of combinations was chosen by increasing the number of tests until 10 consecutive tests' optimal values varied within $<1 \%$. By computing the design factor, we were able to evaluate the design factor's global maxima within the feasible design parameters intervals. By plotting the design factor against each parameter, we were able to detect how each parameter affects the design factor. We were then able to find analytic ratios of different parameters that led to an optimal design factor, previously found by the numerical search. We applied these ratios to perform the analytical analysis. We substituted these best performing parameter ratios as limits to the analytic expression for the tri-layer curvature, Equation (10). Subsequently, we were able to optimize the new substituted expressions analytically. These optimal expressions were able to reproduce the global numerical optimal values within a reasonable accuracy, as will be discussed in Summary section. In a trilayer design with a single conductive layer, the conductive layer can be either one of the outer layers, or the middle layer. In this section, we optimize each case separately to find the best overall configuration.

Outer conductive layer. We begin by optimizing the case where the conductive layer is on either one of the outer layers. For convenience, without loss of generality, we will refer to the conductive layer as layer 1 and, therefore, mark the nonconductive outer as layer 3 . We can rewrite Equation (10) for $i=1$ using dimensionless parameterization $t_{21}=t_{2} / t_{1}, t_{31}=t_{3} / t_{1}, \quad E_{21}=E_{2} / E_{1}, \quad E_{31}=E_{3} / E_{1}$ as

\section{Table 2. Feasible Design Parameters Values} FOR THE NumERICAL ANALySIS

\begin{tabular}{ll}
\hline$t_{1,2,3}(\mu \mathrm{m})$ & {$[1,100]$} \\
$E_{1,2,3}(\mathrm{~Pa})$ & {$\left[10^{4}, 10^{10}\right]$} \\
$\alpha_{1,2,3}\left(\mathrm{~K}^{-1}\right)$ & {$\left[10^{-6}, 10^{-4}\right]$} \\
\hline
\end{tabular}




$$
\kappa=\frac{C V^{2}}{\rho} \cdot \frac{w}{l} \cdot \frac{2\left(\left(\alpha_{2}-\alpha_{3}\right)\left(t_{21}+t_{31}\right)+\frac{\left(\alpha_{1}-\alpha_{3}\right)\left(1+2 t_{21}+t_{31}\right)}{E_{21} t_{21}}+\frac{\left(\alpha_{1}-\alpha_{2}\right)\left(1+t_{21}\right)}{E_{31} t_{31}}\right)}{\left(t_{21}+t_{31}\right)^{2}+\frac{\left(1+2 t_{21}+t_{31}\right)^{2}}{E_{21} t_{21}}+\frac{\left(1+t_{21}\right)^{2}}{E_{31} t_{31}}+\frac{\left(1+E_{2} t_{21}{ }^{3}+E_{3} t_{31}^{3}\right)\left(1+E_{2} t_{21}+E_{3} t_{31}\right)}{3 E_{21} E_{31} t_{21} t_{31}}} .
$$

We used the numerical method, explained earlier, to map the design factor within the intervals in Table 2 and found that for $E_{21} t_{21} \gg 1$ and $E_{31} t_{31} \gg 1$ the design factor simplifies to

$$
\frac{2\left(\left(\alpha_{2}-\alpha_{3}\right)\left(t_{21}+t_{31}\right)\right)}{\left(t_{21}+t_{31}\right)^{2}+\frac{\left(1+E_{21} t_{21}{ }^{3}+E_{31} t_{31}{ }^{3}\right)\left(1+E_{21} t_{21}+E_{31} t_{31}\right)}{3 E_{21} E_{31} t_{21} t_{31}}} .
$$

For small enough values of $t_{21}$ and $t_{31}$ we can achieve $E_{21} t_{21}^{3} \ll 1$ and $E_{31} t_{31}^{3} \ll 1$ while keeping our previous demands of $E_{21} t_{21} \gg 1$ and $E_{31} t_{31} \gg 1$. For these values the sum $E_{21} t_{21}{ }^{3}+E_{31} t_{31}{ }^{3}$ can then be neglected and the design factor expression can be further simplified to

$$
\frac{2\left(\alpha_{2}-\alpha_{3}\right)}{t_{21}+t_{31}}
$$

Substituting the simplified design factor expression Equation (13) into Equation (11) we arrive at the final simplified curvature expression

$$
\kappa=\frac{C V^{2}}{\rho} \cdot \frac{w}{l} \cdot 2 \frac{\alpha_{2}-\alpha_{3}}{t_{21}+t_{31}} .
$$

This result is similar to the optimal external heating bilayer expression [Equation (5)], it is proportional to the CTE difference of both of the nonconductive layers and can potentially achieve unbounded values. For a set of materials that satisfy $E_{21} t_{21} \gg 1, E_{31} t_{31} \gg 1, E_{21} t_{21}^{3} \ll 1, E_{31} t_{31}^{3} \ll 1$ as $t_{21}, t_{31} \rightarrow 0$, the curvature sensitivity to voltage will approach infinity. This optimal tri-layer, with outer conductive layer, is essentially an optimal bi-layer with a thick and soft conductive outer layer. The thick and soft conductive layer provides an effective voltage-to-temperature conversion while keeping the former optimal externally heated bi-layer performance.

Middle (inner) conductive layer. We finalize by optimizing the case of the tri-layer when the mid-layer is the conductive heating source. We can write Equation (10), for $i=2$, with the following dimensionless parameterizations $t_{12}=t_{1} / t_{2}, \quad t_{32}=t_{3} / t_{2}, \quad E_{12}=E_{1} / E_{2}, \quad E_{32}=E_{3} / E_{2}$ resulting in
The conductive middle layer design is symmetric, switching between the outer nonconductive layers is equivalent to turning over the actuator and, therefore, there are two equivalent optimal solutions. We will present one of the symmetric optimal solutions, the other equivalent optimal solution can be obtained by switching the indices 1 with 3 . The optimization was done using the same method as in the outer-layer case. The optimal design factor was found for $E_{12} t_{12} \gg 1 \gg E_{32} t_{32}$, where it can be approximated as

$$
\frac{2\left(\alpha_{1}-\alpha_{2}\right)\left(t_{12}+1\right)}{\left(t_{12}+1\right)^{2}+\frac{\left(E_{12} t_{12}{ }^{3}+1+E_{32} t_{32}^{3}\right)}{3}} .
$$

As in the outer-layer case, for small enough values of $t_{12}$, $E_{12} t_{12}^{3} \ll 1$ the optimal design factor can be further approximated to a simpler expression

$$
\frac{6\left(\alpha_{1}-\alpha_{2}\right)}{3\left(t_{12}+1\right)+1} .
$$

We can substitute this simplified approximated optimal design factor [Equation (17)] into the curvature expression (Equation (15)] to arrive at the final simplified optimal curvature expression

$$
\kappa=\frac{C V^{2}}{\rho} \cdot \frac{w}{l} \cdot \frac{6\left(\alpha_{1}-\alpha_{2}\right)}{3\left(t_{12}+1\right)+1} .
$$

This result is similar to the internally heated bi-layer case in Equation (5). It is proportional to the CTE difference of both of the nonconductive layers and its design factor can only achieve a bound value. In the conductive mid-layer case, as in the outer-layer case, the optimal design factor is proportional to the nonconductive outer layers CTE difference. However, unlike the outer-layer case, the design factor is bounded. For $E_{12} t_{12} \gg 1 \gg E_{32} t_{32}$ and $E_{12} t_{12}^{3} \ll 1$ as $t_{12} \rightarrow 0$, the curvature approaches $\kappa=\frac{C V^{2}}{\rho} \cdot \frac{w}{l} \cdot \frac{3}{2}\left(\alpha_{1}-\alpha_{3}\right)$. This optimization, with optimal parameters, can only achieve the same curvature sensitivity as the optimal internally heated bi-layer.

\section{Summary}

In this study, we developed, optimized, and experimentally validated the bi- and tri-layer analytical models. The models

$$
\kappa=\frac{C V^{2}}{\rho} \cdot \frac{w}{l} \cdot \frac{2\left(\frac{\left(\alpha_{2}-\alpha_{3}\right)\left(1+t_{32}\right)}{E_{12} t_{12}}+\left(\alpha_{1}-\alpha_{3}\right)\left(t_{12}+2+t_{32}\right)+\frac{\left(\alpha_{1}-\alpha_{2}\right)\left(t_{12}+1\right)}{t_{12} E_{32}}\right)}{\frac{\left(1+t_{32}\right)^{2}}{E_{12} t_{12}}+\left(t_{12}+2+t_{32}\right)^{2}+\frac{\left(t_{12}+1\right)^{2}}{E_{32} t_{32}}+\frac{\left(E_{12} t_{12}{ }^{3}+1+E_{32} t_{32}^{3}\right)\left(t_{12} E_{12}+1+t_{32} E_{32}\right)}{3 E_{12} E_{32} t_{12} t_{32}}} .
$$


Table 3. Theoretical and Numerical Optimal Design Factor Values Results of the Internally Heated Models

\begin{tabular}{lcc}
\hline & $\begin{array}{c}\text { Approximated optimal } \\
\text { design factor value }\end{array}$ & $\begin{array}{c}\text { Numerical design } \\
\text { factor optimization value }\end{array}$ \\
\hline Internal heated bi-layer $\left(\frac{1}{{ }^{\circ} \mathrm{C}}\right)$ & $1.5 \times \frac{\alpha_{2}-\alpha_{1}}{t_{12}+1}=1.4703 \times 10^{-4}$ & $1.44 \times 10^{-4}$ \\
Internal heated tri-layer $\left(\frac{1}{{ }^{\circ} \mathrm{C}}\right)$ (outer conductive layer) & $2 \times \frac{\alpha_{2}-\alpha_{3}}{t_{31}+t_{21}}=9.9 \times 10^{-3}$ & $4.9 \times 10^{-3}$ \\
Internal heated tri-layer $\left(\frac{1}{{ }^{\circ} \mathrm{C}}\right)$ (mid conductive layer) & $\frac{6\left(\alpha_{1}-\alpha_{2}\right)}{3\left(t_{12}+1\right)+1}=1.474 \times 10^{-4}$ & $1.44 \times 10^{-4}$ \\
\hline
\end{tabular}

were investigated for optimal design concerning the design parameters $t_{1,2,3}, E_{1,2,3}, \alpha_{1,2,3}$ and the arrangement of layers. The optimization objective is to find the maximal curvature input sensitivity to either voltage or temperature change. We found that for the more common bi-layer design, the externally and internally heated cases optimal value varies dramatically. For the case of external heating (as in sensors), the optimal design [Equation (5)] requires the layers to be as thin as possible. This design performance, that is, curvature per temperature change, is potentially unbounded. For a given difference in CTE and layer elasticity ratio, the optimal performance is bounded only by the manufacturing capabilities of the layers' thicknesses. For an elasticity ratio of $E_{21}=t_{21}{ }^{2}$, the thinner the layers, the better the performance $\left[\kappa_{\text {opt bi ext }} \propto 1 /\left(t_{1}+t_{2}\right)\right]$. However, the optimal internal heating (ETA) bi-layer performance, that is, curvature-to-voltage ratio [Equation (5)], for a given CTE difference, electrical conductivity, width, and length, is bounded regardless of the other design parameters values $\left[\kappa_{\text {opt bi int }} \propto 1 /\left(t_{21}+1\right)\right]$. This significant difference in performance is due to a "conflict of interest." The optimal externally heated bi-layer design conflicts with the internal heating requirement for low electrical resistance and, therefore, cannot achieve the same performance. The tri-layer design, however, can bypass this conflict by adding a relatively soft and thick conductive layer. An added soft and thick layer can have an excellent voltage-to-
FIG. 8. An FIB cross-sectional image of an actuator with CNT layer thickness of $1.90 \pm 0.2 \mu \mathrm{m}$. CNT, carbon nanotube; FIB, focused ion beam.
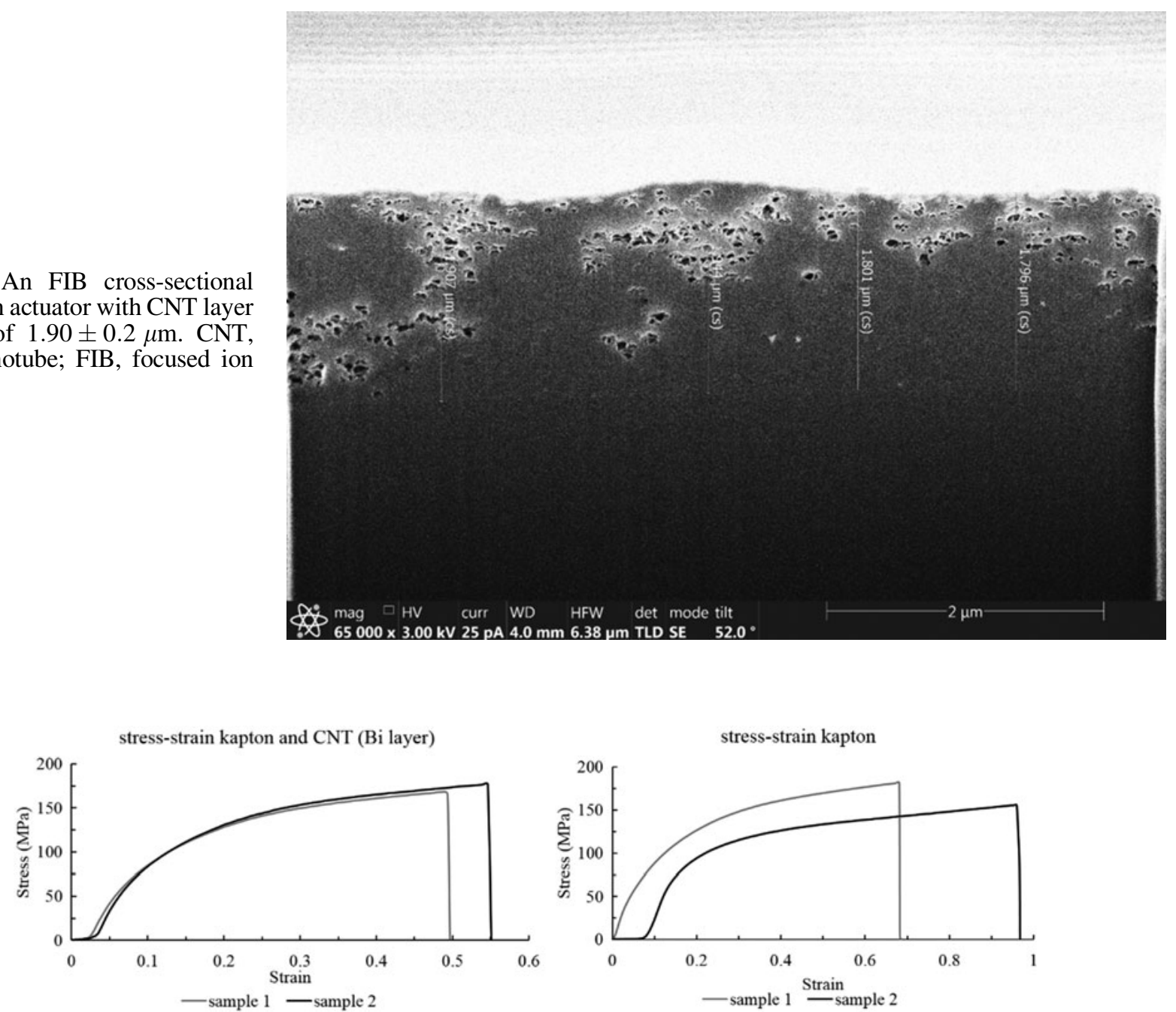

FIG. 9. The stress-strain curves of two tests for Kapton (right) and a bi-layer Kapton-CNT (left). 

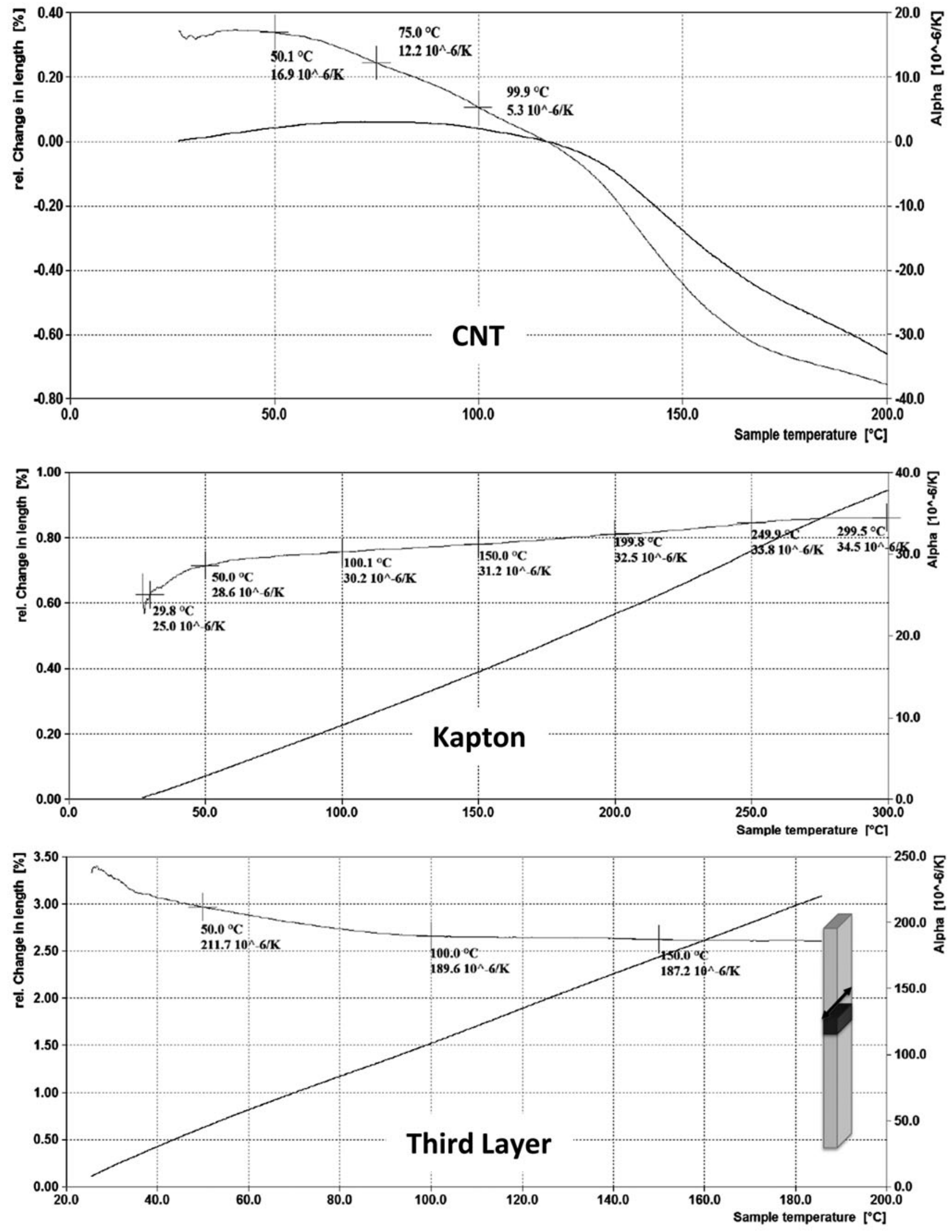

FIG. 10. An optical dilatometer CTE and elongation reading of each layer material. CTE, coefficients of thermal expansion. 
temperature ratio while not impairing the other two layers' passive bi-layer mechanism.

Two tri-layer configurations with internal heating were investigated, a mid-layer conductive layer and an outer conductive layer. We found the optimal parameter ratios required for each of the internally heated cases and their approximate curvature expressions [Equations (5), (14), and (18)]. These parameter ratios provide the maximal temperature-tocurvature ratio and thus represent the performance of the internally heated actuator. Out of all the internally heated cases, the best performing case was found to be the one with the outer conductive tri-layer. The optimal parameters for the outer conductive tri-layer must satisfy $E_{21} t_{21} \gg 1, \quad E_{31} t_{31} \gg 1$, $E_{21} t_{21}^{3} \ll 1$, and $E_{31} t_{31}^{3} \ll 1$ as shown in the Tri-Layer Optimization section. Using these conditions, the optimal tri-layer with outer conductive layer is essentially an optimal externally heated bi-layer with a thick and soft conductive outer layer. The thick and soft conductive layer provides an effective voltage-to-temperature conversion while keeping the former optimal externally heated bi-layer performance.

To test our approximations' assumptions in Equations (5), (14), and (18), we numerically evaluated the optimal design factor of each of the externally heated cases original expressions without assumptions [in Equations (4), (11), and (15)]. For the numerical optimization, we randomly picked a large number of different design parameters combinations, $t_{1,2,3}, E_{1,2,3}, \alpha_{1,2,3}$, from within the intervals in Table 2. The optimal performance numerical search was bounded within a set of feasible design parameters intervals to test the approximation's validity. The feasible elasticity and CTE intervals were chosen based on commonly used engineering materials (as found e.g., in Refs. ${ }^{6}$ and ${ }^{40}$ ). The feasible thickness interval was chosen arbitrarily based on our manufacturing capabilities. For each random material combination, we computed the design factor's numerical value from the relevant expression. The comparative results are summarized in Table 3. Out of the three internal heating cases, the best performing case was the tri-layer with outer conductive layer, as predicted in the Tri-Layer Optimization section. As can be seen in the second row of Table 3, the optimal outer conductive layer case achieved a significant improvement in performance over all the other internally heated designs. The outer layer optimal value approximation has predicted an improvement in performance of $>6000 \%$, whereas the nonapproximated numerical optimization had achieved only a $3000 \%$ improvement. Since the approximated optimal value of Equation (14) requires that $E_{21} t_{21} \gg 1, \quad E_{31} t_{31} \gg 1, \quad E_{21} t_{21}^{3} \ll 1$, and $E_{31} t_{31}^{3} \ll 1$, within the parameter intervals in Table 2, we can only approach these conditions to a certain extent. Although the theoretical approximated optimal value is not realistically achievable, the bounded numerical optimization achieved the aforementioned performance improvement, which is approximately half of the theoretical value. These results emphasize the merits of the tri-layer design and may serve the designers of soft ETA-based robots and provide a superior design to the commonly used bi-layer actuator.

To summarize, in this study we improved upon the common ETA bi-layer design, established a reliable ETA tri-layer model and found the optimal parameters and layer arrangement for minimal voltage-per-curvature change. We intend to utilize the tri-layer outer conductor design to design low-voltage soft thin worm-like locomoting robots (as shown in Fig. 1). We intend to further investigate actuating two-dimensional sheet-like structures that utilize origami folds behavior. Large sheet actuation opens new questions about deformations in two directions, which we will attend to in the future.

\section{Acknowledgments}

We thank Alon Danay for the experimental setup and also express our gratitude to TA instruments for performing the CTE measurements.

\section{Author Disclosure Statement}

No competing financial interests exist.

\section{Funding Information}

This research was partially supported by the Singapore National Research Foundation under the CREATE program: Nanomaterials for Energy and Water-Energy Nexus and by the Israeli Ministry of Defense.

\section{References}

1. Jones BA, Walker ID. Kinematics for multisection continuum robots. IEEE Trans Robot 2006;22:43-55.

2. Robinson G, Davies JBC. Continuum robots-a state of the art. In Proceedings 1999 IEEE International Conference on Robotics and Automation. Detroit, MI: IEEE, 1999, Vol. 4, pp. 2849-2854.

3. Zhou Z, Yan Q, Liu C, et al. An arm-like electrothermal actuator based on superaligned carbon nanotube/polymer composites. New Carbon Mater 2017;32:411-418.

4. Ho-Yin C, Li WJ.A thermally actuated polymer micro robotic gripper for manipulation of biological cells. In IEEE International Conference on Robotics and Automation. Taipei, Taiwan: IEEE, 2003, Vol. 1, pp. 288-293.

5. Shian S, Bertoldi K, Clarke DR. Dielectric elastomer based 'grippers' for soft robotics. Adv Mater 2015;27: 6814-6819.

6. Majidi C. Soft robotics: a perspective-current trends and prospects for the future. Soft Robot 2014;1:5-11.

7. Peraza-Hernandez EA, Hartl DJ, Malak RJ, et al. Origamiinspired active structures: a synthesis and review. Smart Mater Struct 2014;23.

8. Okuzaki H, Saido T, Suzuki H, et al. A biomorphic origami actuator fabricated by folding a conducting paper. J Phys Conf Ser 2008;127:012001.

9. Rus D, Tolley MT. Design, fabrication and control of soft robots. Nature 2015;521:467-475.

10. McMahan W, Bryan J, Ian W, et al. Robotic manipulators inspired by cephalopod limbs. CDEN Design Conference. McGill University, Montreal, Quebec: Canadian Engineering Education Association (CEEA), 2004, pp. 1-10.

11. Shapiro Y, Wolf A, Gabor K. Bi-bellows: pneumatic bending actuator. Sens Actuator A Phys 2011;167:484-494.

12. Matia Y, Gat AD. Dynamics of elastic beams with embedded fluid-filled parallel-channel networks. Soft Robot 2015;2:42-47.

13. Shepherd RF, Ilievski F, Choi W, et al. Multigait soft robot. Proc Natl Acad Sci 2011;108:20400-20403.

14. Shepherd RF, Stokes AA, Freake J, et al. Using explosions to power a soft robot. Angew Chem Int Ed 2013; 52:2892-2896. 
15. Seo DK, Kang TJ, Kim DW, et al. Twistable and bendable actuator: a CNT/polymer sandwich structure driven by thermal gradient. Nanotechnology 2012;23:075501.

16. Albu-Schäffer A, Antonio B. Springer handbook of robotics. In Springer Handbook of Robotics, Bruno Siciliano OK. (Ed). Berlin, Germany: Springer International Publishing, 2016, pp. 499-530.

17. Onal CD, Wood RJ, Rus D. Towards printable robotics: origamiinspired planar fabrication of three-dimensional mechanisms. In 2011 IEEE International Conference on Robotics and Automation. Shanghai, China: IEEE, 2011, pp. 4608-4613.

18. Shigemune H, Maeda S, Hara Y, et al. Design of paper mechatronics: towards a fully printed robot. In 2014 IEEE/ RSJ International Conference on Intelligent Robotics and Systems, 2014, pp. 536-541.

19. Shin B, Ha J, Lee M, et al. Hygrobot: a self-locomotive ratcheted actuator powered by environmental humidity. Sci Robot 2018;3:eaar2629.

20. Sellinger AT, Wang DH, Tan LS, et al. Electrothermal polymer nanocomposite actuators. Adv Mater 2010;22: 3430-3435.

21. Hu Y, Chen W, Lu L, et al. Electromechanical actuation with controllable motion based on a single-walled carbon nanotube and natural biopolymer composite. ACS Nano 2010;4:3498-3502.

22. Hu Y, Wang G, Tao X, et al. Low-voltage-driven sustainable weightlifting actuator based on polymer-nanotube composite. Macromol Chem Phys 2011;212:1671-1676.

23. Yao S, Cui J, Cui Z, et al. Soft electrothermal actuators using silver nanowire heaters. Nanoscale 2017;9:37973805 .

24. Chen L, Liu C, Liu K, et al. High-performance, lowvoltage, and easy-operable bending actuator based on aligned carbon nanotube/polymer composites. ACS Nano 2011;5:1588-1593.

25. Hou MT, Chen R. Effect of width on the stress-induced bending of micromachined bilayer cantilevers. J Micromech Microeng 2003;13:141-148.

26. Chen L, Weng M, Zhou Z, et al. Large-deformation curling actuators based on carbon nanotube composite: advancedstructure design and biomimetic application. ACS Nano 2015;9:12189-12196.

27. Li Q, Liu C, Lin Y, et al. Large-strain, multiform movements from designable electrothermal actuators based on large highly anisotropic carbon nanotube sheets. ACS Nano 2015;9:409-418.

28. Lau PH, Takei K, Wang C, et al. Fully printed, high performance carbon nanotube thin-film transistors on flexible substrates. Nano Lett 2013;13:3864-3869.
29. Erdem EY, et al. Thermally actuated omnidirectional walking microrobot. J Microelectromech Syst 2010;19:433-442.

30. Timoshenko SP. Analysis of bi-metal thermostats. J Opt Soc Am 1925;11:233-255.

31. Kanthal Corporation. Kanthal Thermostatic Bimetal Handbook. Hallstahammar, Sweden: Bulten-Kanthal AB, 2008.

32. Vasudevan M, Johnson W. On multi-metal thermostats. Appl Sci Res Sect B 1963;9:420-430.

33. Shapiro B, Smela E. Bending actuators with maximum curvature and force and zero interfacial stress. J Intell Mater Syst Struct 2007;18:181-186.

34. Benslimane M, Gravesen P, West K, et al. Performance of polymer-based actuators: the three-layer model. In 1999 Symposium on Smart Structures and Materials, Vol. 3669. Newport Beach, CA: SPIE, 1999, pp. 87-97.

35. Balakrisnan B, Nacev A, Smela E. Element method design of bending multi-layer electroactive polymer actuators. Smart Mater Struct 2015;24:045032.

36. Tibi G, Sachyani E, Layani M, et al. Analytic modeling and experiments of tri-layer, electro-thermal actuators for thin and soft robotics. In Proceedings of the IEEE International Conference on Robotics and Automation. Singapore, Singapore: IEEE, 2017.

37. Sachyani E, Layani M, Tibi G, et al. Enhanced movement of CNT-based actuators by a three-layered structure with controlled resistivity. Sens Actuator B Chem 2017;252: 1071-1077.

38. Wang C, Nosaka T, Yost B, et al. Printed carbon nanotubes on polymer films for active origami. Mater Res Lett 2013;1: 13-18.

39. Gerratt AP, Balakrisnan B, Penskiy I, et al. Design of bending multi-layer electroactive polymer actuators. Smart Mater Struct 2015;24.

40. Ahrens TJ, ed. Mineral Physics \& Crystallography: A Handbook of Physical Constants, Vol. 2. Washington, DC: American Geophysical Union, 1995.

Address correspondence to: Amir Degani

Department of Environmental, Water and Agricultural Engineering

Faculty of Civil and Environmental Engineering Technion Israel Institute of Technology 609 Rabin Building Haifa 32000 Israel

E-mail: adegani@technion.ac.il

\section{Appendix}

\section{Fabrication Information}

\section{Carbon nanotube ink preparation}

Highly concentrated multiwalled carbon nanotube (MWCNT) inks were prepared mixing $1 \mathrm{wt} \%$ of MWCNTs, NC7000TM (Nanocyl S.A., Belgium) a polymeric dispersant SOLSPERSE $^{\circledR} 46000$ (2 wt\%; Lubrizol), and a wetting agent (0.1 wt\%, Byk 348; Bykchemie GmbH, Germany), with deionized water. The ink was prepared using a horn sonicator (Vibracell; Sonics \& Materials, Inc.) for $30 \mathrm{~min}$ at 637.5 W. The samples were cooled in an ice water bath during the sonication process. After sonication, the ink was further concentrated by lyophilization using a freeze-dry system (Free Zone 2.5; Labconco Corp.) for $4 \mathrm{~h}$ under pressure of $<1$ mbar. The final carbon nanotube (CNT) concentration was $\sim 1.3 \mathrm{wt} \%$. 


\section{Ink deposition}

The CNT ink was deposited on the substrate using a drawdown method. Drawdown was performed using a K101 Control Coater with an adjustable micrometer applicator (RK PrintCoat Instruments Ltd., United Kingdom). Four layers were deposited with a micrometer applicator, 50 nominal scale of the bar coater, and each wet layer was dried using a hot air gun before the next layer was applied. The CNT ink was deposited onto a commercial Kapton film (200 HN; Dupont) having dimensions of $2 \times 6 \mathrm{~cm}$, and film thickness of $50.8 \mathrm{~m}$.

\section{Formation of third layer}

UV curable ink was formed composed of aliphatic urethane acrylate monomers (CN9900; Sartomer, Belgium). A photoinitiator [Diphenyl(2,4,6-trimethylbenzoyl)phosphine oxide, TPO; BASF, Germany] was dissolved in the monomers, at $80^{\circ} \mathrm{C}$. The deposition of the third layer was done by drawdown of the UV curable ink onto the bi-layer actuator, followed by UV light radiation (P1, Portable UV LED Curing System; $395 \pm 5 \mathrm{~nm}$, maximum intensity $8 \mathrm{~W} / \mathrm{cm}^{2}$ ). In experiments with different thicknesses of the third layer, the layers were drawdown using a K101 Control Coater with an adjustable micrometer applicator (RK PrintCoat Instruments Ltd.).

\section{Characterization}

\section{Movement measurements}

The actuator was connected to electrodes by means of silver paste and copper tape. Movements were measured by applying constant voltage by a function generator (TG315; AimTTi, United Kingdom) and a Signal Amplifier (9100A, 400Vp-p Single-Channel; Tabor Electronics Ltd., Israel). Photos of the actuators in movement were taken by a USB microscope camera (AM4000 Series; Dino-Lite, Taiwan). Determination of the curvature was performed using the Dinocapture 2.0 software.
The temperature of the actuator was measured using a thermal camera (E4; FLIR). The maximal temperature is defined as the temperature reached at the spot on the actuator with the highest temperature at a given constant voltage. This can be read both manually, or automatically, by the FLIR instrument.

\section{Thickness evaluation}

Thickness evaluations of the CNT layer were performed by cross-sectional measurements of the samples, using a DualBeam Focused Ion Beam (FIB)/SEM system (Helios NanoLab 460F1; FEI) (example shown in Fig. 8).

\section{Elasticity measurements}

Mechanical properties were measured by performing a tensile test on actuator samples using a Universal Testing Machine (Instron 4500; Instron) with $500 \mathrm{~N}$ load cell, tension rate of $10 \mathrm{~mm} / \mathrm{min}$, and initial distance of $20 \mathrm{~mm}$ between grippers. Since we were unable to measure the CNT layer separately we measured a bi-layer sample of Kapton (Fig. 9, right) and CNT (Fig. 9, left) and evaluated the CNT's young modulus. The third layer elasticity measurement is more elaborate and is discussed in our previous study. ${ }^{\text {A1 }}$

\section{CTE measurements}

CTE of polymer films and CNT layers were measured by an optical dilatometer DILs 806 (TA Instruments) with heat rate of $2^{\circ} \mathrm{C} / \mathrm{min}$ and temperatures ranging from room temperature to $200^{\circ} \mathrm{C}$. The results are depicted in Figure 10 .

\section{Appendix Reference}

A1. Sachyani E, Layani M, Tibi G, et al. Enhanced movement of CNT-based actuators by a three-layered structure with controlled resistivity. Sens Actuator B Chem 2017;252: 1071-1077. 